\title{
Entropic Analysis of Color Homogeneity
}

Kirill Alemaskin

Case Western Reserve University

Ica Manas Zloczower

Case Western Reserve University, ica.manas-zloczower@case.edu

Miron Kaufman

Cleveland State University, m.kaufman@csuohio.edu

Follow this and additional works at: https://engagedscholarship.csuohio.edu/sciphysics_facpub

Part of the Physics Commons

How does access to this work benefit you? Let us know!

Publisher's Statement

This is the accepted version of the following article: Kirill Alemaskin, Ica Manas-Zloczower and Miron Kaufman, "Entropic analysis of color homogeneity," Polymer Engineering \& Science 45 (7), 1031-1038 (2005). , which has been published in final form at http://onlinelibrary.wiley.com/ wol1/doi/10.1002/pen.20320/abstract

\section{Repository Citation}

Alemaskin, Kirill; Zloczower, Ica Manas; and Kaufman, Miron, "Entropic Analysis of Color Homogeneity" (2005). Physics Faculty Publications. 234.

https://engagedscholarship.csuohio.edu/sciphysics_facpub/234

This Article is brought to you for free and open access by the Physics Department at EngagedScholarship@CSU. It has been accepted for inclusion in Physics Faculty Publications by an authorized administrator of

EngagedScholarship@CSU. For more information, please contact library.es@csuohio.edu. 


\title{
Entropic Analysis of Color Homogeneity
}

\author{
Kirill Alemaskin, Ica Manas-Zloczower \\ Miron Kaufman
}

\section{INTRODUCTION}

Color homogeneity is an important aspect of quality and consumer satisfaction in a variety of sectors. Examples are automotive, textiles, food, and packaging, to mention just the most prominent in the plastics and rubber industry. One commonly used method to assess color homogeneity is comparison to a standard for qualitative visual inspection. Color homogeneity can also be assessed by using spectroscopic methods. When colored samples are obtained from an experiment, they can be analyzed with particular-type spectrometers to give color coordinates and/or other color characteristics of the sample. Color homogeneity can also be assessed by using computer image analysis and employing standard direct red-blue-green (RGB) correlations in the studied sample. Translating the results into a quantitative index of color homogeneity to be employed in process control and optimization is not an easy task.

Conversely, color homogeneity or, in more general terms, property homogeneity, is achieved through mixing. Thus color homogeneity assessment may become a tool in evaluating mixing efficiency in processing and equipment design optimization.

As an example we consider mixing and in particular distributive mixing evaluation in an extrusion process. Be- pining an important component of the extrusion process, mixing evaluation and optimization has been the focus of ex- feat tensive research over the years. In an extruder, mixing distributive mixing devices for single screw extruders deoccurs from the solid conveying zone to the exit from the veloped over the years designed primarily to impart subdie. Assessing mixing along the extruder length remains a stantial shear strain and also provide material reorientation challenge.
Maddock [1] in the late 1950s proposed a technique of visual screw mixing analysis, known as "screw freezing" or "screw crash," which has been extensively used since its inception. Benkreira et al. [2] showed that mixing primarily occurs during the melting phase of extrusion, and little improves thereafter. In a conventional Archimedean screw, during the melting stage, breakage of the solid bed in the cross channel direction can occur. This creates solid fragments that flow downstream not melted, thus reducing mixing quality [3]. To overcome this problem, several screw designs have been proposed, including the barrier melting screws $[4,5]$ and Maddock-style mixers [6, 7]. Other extruder designs implement the idea of forcing the material to flow through regions with repeatedly changing clearances, such as the energy transfer (ET) screws [8] or the Stratablend mixing screw [9]. These designs are mostly useful in enhancing melting efficiency and distributive mixing of the screw.

Many fundamental analyses of mixing in extrusion have focused on the melt conveying zone [10-15]. While conventional screws do not provide high mixing efficiency, the application of mixers designed to reorient the flow streamlines and improve mixing in the metering zone, such as pin-type mixers [16-19], or the introduction of spatially periodic barriers in the screw to generate chaotic flow features [20], enhance mixing. There is a large number of [21-27].

Mixing continues past the metering zone of the extruder as the polymer flows through the die. Special dies have been 
designed to promote mixing, with one example being the spiral mandrel die for annular products [28, 29].

Mixing characterization and quantification is not an easy task and still poses many challenges. Among the measures most frequently used to assess the degree of mixing in a system are gross uniformity and intensity of segregation [30], scale of segregation [31], and striation thickness [32]. Prediction of minor component spatial distribution inside the extruder by using a particle tracking technique in numerical simulations allows mixing characterization through pairwise correlation functions [33, 34]. More recently, Renyi entropies have been employed as a rigorous measure of distributive mixing in polymer processing equipment $[35,36]$.

Entropic measures are being applied in many fields of science such as statistical mechanics [37, 38], biology and medicine ]39-41], cognitive science [42, 43], economics [44, 45], and geology [46, 47], to list just a few examples. In pattern recognition, Cheng et al. [48, 49] introduced the entropy-based fuzzy homogeneity approach and the method of homograms applied to image threshold and segmentation. Entropic image analysis has also been used to assess the level of image compression, as was shown by Tavakoli [50].

In this work we propose an entropic measure to assess color homogeneity and deviations from an ideal color and employ this measure as a tool to assess distributive mixing efficiency in a single screw extruder. Entropy is the rigorous measure of disorder or system homogeneity. Since we are interested in measuring the degree of distributive mixing achieved in the equipment, we propose to use entropy to quantify color homogeneity as a measure of distributive mixing and furthermore employ it as a tool for equipment design optimization. The information (Shannon) entropy defined in Eq. 1 below is the standard measure [51] of homogeneity as it is uniquely determined by the following common-sense (Khinchin) requirements [52]: (i) the lowest entropy corresponds to one of the probabilities being unity and the rest being zero (i.e., total information, perfect order, highest inhomogeneity); (ii) the largest value for the entropy is achieved when all probabilities are equal to each other (i.e., the absence of any information, complete disorder, perfect homogeneity); and (iii) the entropy is additive over partitions of the outcomes. One important consequence of introducing the entropic measure, which is the central concept of information theory, into the field of color characterization is the linkage of this applied engineering field to rigorous methods employed in the rest of the sciences and engineering.

\section{BACKGROUND}

Entropy is the rigorous measure of mixing. The information entropy for a particular experimental condition with a set of $\mathrm{M}$ possible outcomes is:

$$
S=-\sum_{i=1}^{M} p_{i} \ln p_{i}
$$

where $p_{i}$ is the probability of occurrence of outcome $i$ [51].

When assessing the particle distribution of a minor component throughout a polymer matrix by employing entropy, we divide our domain of interest (which can be two- or three-dimensional) into $M$ equal size regions (bins) and then calculate particle concentrations in each bin as estimators of the probabilities. For a certain number of bins $M$, maximum entropy is reached if particle concentrations are equal in all bins (particles are evenly distributed throughout the system-ideal distribution). This maximum has a value of $\ln$ $M$. Thus an index of system homogeneity can be obtained by dividing the information entropy by $\ln M$. Shannon called this index a relative entropy.

$$
S_{\text {rel }}=\frac{S}{\ln (M)}
$$

In previous work $[35,36,53]$ we have shown that the number of bins chosen to describe the quality of distributive mixing is an important parameter as it defines the scale of observation (the "magnifying glass") at which one looks at the system. The smaller the number of bins, the larger the scale of observation at which the observer evaluates the quality of particle distribution. At a very small scale $(M$ $>\mathrm{N}$ ) some bins will be depleted of particles and the particle distribution will thus differ from the uniform distribution. Furthermore, to estimate probabilities by using frequencies, we need large numbers of particles. Thus the number of particles in the system sets an upper limit for the number of bins to be used for system characterization.

When more than one minor component species is present in the system, for instance particles of different colors, the system entropy can be calculated by using joint probabilities $p_{c, j}$ (probability for a particle to be of a color " $c$ " and in bin " $j$ "):

$$
S=-\sum_{c=1}^{C} \sum_{j=1}^{M} p_{c, j} \ln p_{c, j}
$$

with $C$ being the total number of species present in the system.

When using Bayes theorem, the joint probability for a particle to be located in bin $j$ and to be of a color $c$ can be calculated from: $p_{c, j}=p_{c / j} p_{j}$, where $p_{c / j}$ is the probability of finding a particle of a color " $c$ " conditional on the bin " $j$ " and $p_{j}$ is the probability for bin " $j$ ". With that we can write:

$$
S=-\sum_{c=1}^{C} \sum_{j=1}^{M}\left[\left(p_{c / j} p_{j}\right) \ln \left(p_{c l j} p_{j}\right)\right]
$$

It follows:

$$
S=-\sum_{j=1}^{M} p_{j} \sum_{c=1}^{C}\left[p_{c / j} \ln p_{c / j}\right]-\sum_{c=1}^{C} p_{c / j} \sum_{j=1}^{M}\left[p_{j} \ln p_{j}\right]
$$




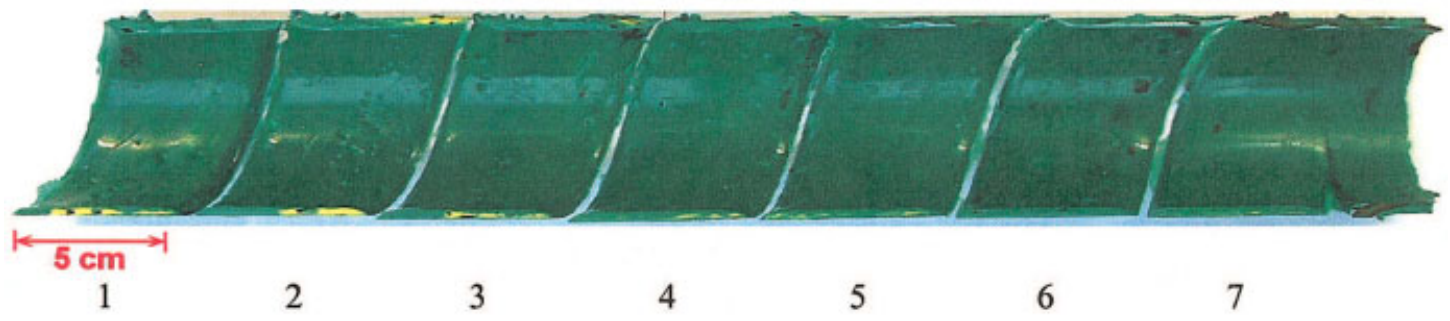

(a)

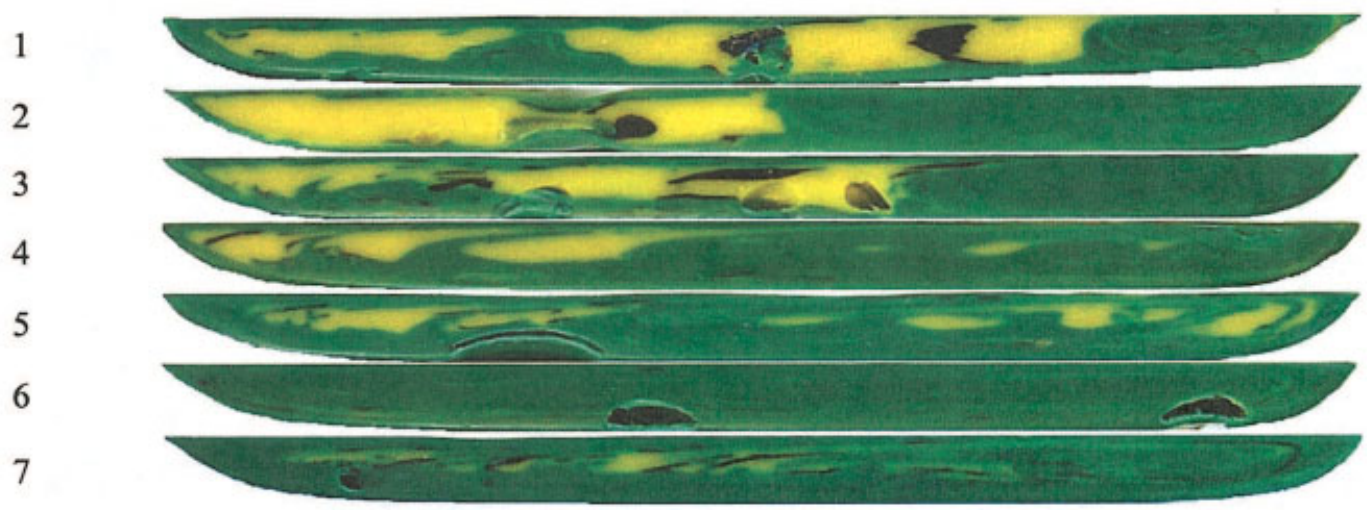

(b)

FIG. 1. (a) Extrusion sample. (b) Cuts analyzed. [Color figure can be viewed in the online issue, which is available at www.interscience.wiley.com.]

and finally because $\sum_{c=1}^{C} p_{c / j}=1$ for all values of $j$

$$
S=\sum_{j=1}^{M}\left[p_{j} S_{j}(\text { species })\right]+S(\text { locations })
$$

where

$$
\begin{aligned}
& S_{j}(\text { species })=-\sum_{c=1}^{C}\left[p_{c / j} \ln p_{c / j}\right] \\
& S(\text { locations })=-\sum_{j=1}^{M}\left[p_{j} \ln p_{j}\right] .
\end{aligned}
$$

$S_{j}$ (species) is the entropy of species intermixing at the location of bin $j$, and $S$ (locations) is the entropy associated with the overall spatial distribution of particles irrespective of colors.

If the species are distinguished by color, Eq. 6 can also be written as:

$$
S=S_{\text {locations }}(\text { colors })+S(\text { locations })
$$

where

$$
S_{\text {locations }}(\text { colors })=\sum_{j=1}^{M}\left[p_{j} S_{j}(\text { colors })\right] .
$$

$S_{\text {locations }}($ colors) is a spatial average of the entropies associated with color intermixing, conditional on location. It is maximized for the particular homogeneous state characterized by $p_{c / j}=1 / C$ in each bin " $j$ " ranging from 1 to $M$. The maximum value of $S_{\text {locations }}$ (colors) is $\ln C$; thus to obtain an index of color homogeneity with values between 0 (no color intermixing) and 1 (ideal intermixing), we normalize this spatial average entropy by $\ln C$.

In the following we will illustrate the use of this index of color homogeneity to analyze extrudate samples obtained by mixing yellow and blue polymeric concentrates in a single screw extruder.

\section{PROCEDURE}

Mixing experiments using pellets of an acrylonitrile butadiene styrene (ABS) resin of two colors (yellow and blue) were performed at Dow Chemical Co. Details of the experimental procedure are described in Ref. 53. Figure 1 shows the extrudate from the "screw crash" experiment and images of 7 cuts obtained in the metering section of the single screw extruder (each corresponding to one turn of the screw). We will analyze the color homogeneity of the 7 
consecutive cuts of the extrudate sample in terms of an index of color homogeneity:

$$
\operatorname{Index}_{\text {color }}=\frac{S_{\text {locations }}(\text { colors })}{\ln C} .
$$

To simplify the analysis, colored pictures can be converted into grayscale images, which in turn can be examined for their homogeneity. In this case the intensity of gray color in each pixel/bin can be considered as a combination of a certain number of white and black particles, the relative concentration of which can be used as estimators of the probabilities [53].

Alternatively, one can analyze the colored pictures by employing the standard red-green-blue (RGB) primary colors at each pixel of the image. Thus by using a digital camera and standard image processing software (e.g., Adobe ${ }^{\circledR}$ Photoshop $^{\circledR}$ ), a matrix of primary color intensities at each pixel of the image can be generated. At a 24-bit image color depth, the intensity for each primary color/channel ranges from 0 to 255 . The channels can be analyzed separately (monochromatic images), with the color intensities interpreted as mixtures of "white" and "black" particles summing up to 255 at each pixel. Thus the intensity of 0 corresponds to 0 "white" and 255 "black" particles, while the intensity of 255 corresponds to 255 "white" and 0 "black" particles. The relative concentrations of "white" and "black" particles serve to calculate the respective probabilities.

The overall color homogeneity will depend on how evenly "black" and "white" particles are distributed throughout the whole image. An equal number of "black" and "white" particles per each pixel means uniform intensity (level of gray) throughout the whole image, which brings the index of color homogeneity to 1 . The maximum number of bins for the calculation will correspond to the number of pixels on the image. Thus at higher image resolutions we can estimate the index of color homogeneity at smaller scales of observation.

Furthermore, we can analyze the images by comparison with an "ideal" color. In the case of gray-scale images this entails choosing a particular combination of black and white particles to obtain the "ideal gray," which is not necessarily obtained at equal concentrations of black and white particles. In the case of color images, we need to choose an "ideal" color translated into different "ideal" intensities for the three RGB channels. In turn, each of these "ideal" intensities is viewed as a combination of "black" and "white" particles summing up to 255. To account for the different black/white ratios in the "ideal" case scenario, we take the $p_{c / j}$ in $E q .7$ (with $c$ standing for black or white) to be:

$$
\begin{aligned}
p_{\text {black } / j}^{*} & =\frac{n_{\text {blacklj }} / n_{\text {ideal black }}}{n_{\text {blacklj } j} / n_{\text {ideal black }}+n_{\text {whitelj } j} / n_{\text {ideal white }}} \\
p_{\text {whitelj }}^{*} & =\frac{\text { and }}{n_{\text {whitelj }} / n_{\text {ideal white }} / n_{\text {blacklj }} / n_{\text {ideal black }}+n_{\text {whitelj }} / n_{\text {ideal white }}}
\end{aligned}
$$

where $n_{\text {black } j}$ and $n_{\text {white/j }}$ are the numbers of "black" and "white" particles in bin $j$ and $n_{\text {ideal black }}$ and $n_{\text {ideal white }}$ are the numbers of "black" and "white" particles corresponding to the ideal combination. One can think of $n_{\text {ideal black }}$ groups of black particles and of $n_{\text {ideal white }}$ groups of white particles, and then Eq. 12 provides the probabilities that such a group in bin $\# j$ is black or white, respectively. Note that the index color is one-that is, maximum - when in each bin the ratio of "black" to "white" particles is the same and is equal to the preferred gray shade:

$$
\frac{n_{\text {blacklj }}}{n_{\text {whitelj }}}=\frac{n_{\text {ideal black }}}{n_{\text {ideal white }}}
$$

Thus the color index defined in Eq. 11 constitutes a simultaneous measure of color homogeneity and of closeness to the "ideal" gray shade.

The cuts shown in Fig. $1 \mathrm{~b}$ include dark regions because of contamination or the presence of voids. In analyzing the images for color homogeneity and deviation from an "ideal" color, one can filter out those defects and the image corners by attributing such regions to "exclusion" intensity.

\section{RESULTS AND DISCUSSION}

Figure 2 shows four sets of pictures representing the converted grayscale and the three split RGB channels (RGB monochromatic intensity maps) of the original color images. To calculate an index of color homogeneity, one can define an "ideal" color or one can choose as the "ideal" the average value for the region of interest. Moreover, the definition of "ideal" can be with respect to the grayscale image or with respect to the color image. In the last case, the definition of "ideal" entails identifying the relative intensities of the split channels that make up for the "ideal" color.

In our analysis we have chosen $n_{\text {ideal white }} / n_{\text {ideal black }}$ combinations to be $70 / 185$ for the converted grayscale image. In the RGB analysis we choose the relative intensities translated as $n_{\text {ideal white }} / n_{\text {ideal black }}$ to be $15 / 240$ for the red split channel, 95/160 for the green split channel, and 25/230 for the blue split channel. It is important to mention that because these combinations were not chosen on the basis of the average values for the entire region of study, the index of color homogeneity does not necessarily reach 1.0 at the largest scale of observation $(M=1)$.

Figure 3 a shows the results obtained by using the grayscale image. The index of color homogeneity shows a general trend of improvement from the first to the last cut, reflecting the color intermixing progression along the extruder line. At small scales of observation (10,000 to 54,000 bins), we observe relative declines in the index of color homogeneity at the 5th and 7th cuts. Examination of the grayscale image (Fig. 2a) reveals a slightly brighter large region on the right hand side of cut \#5 as well as visible non-uniformities for cut \#7. These features of the images do not play a significant role when the analysis is carried out at 


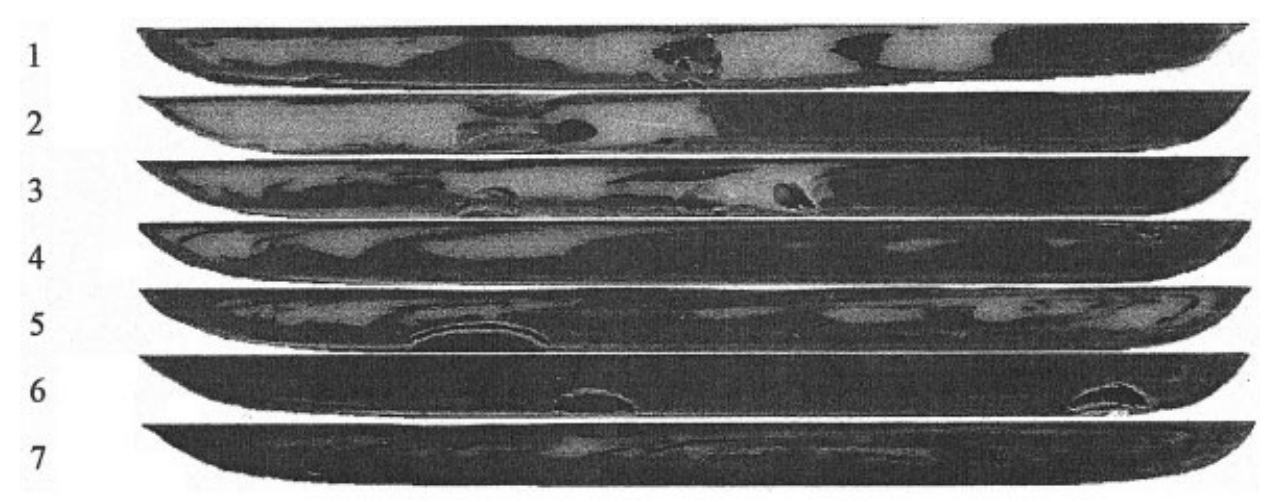

(a)

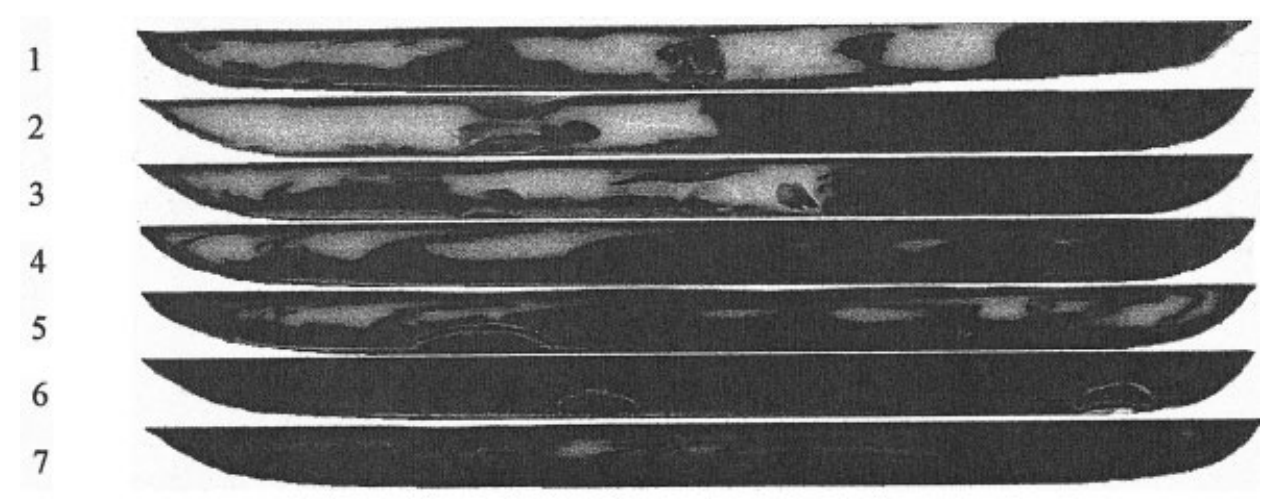

(b)

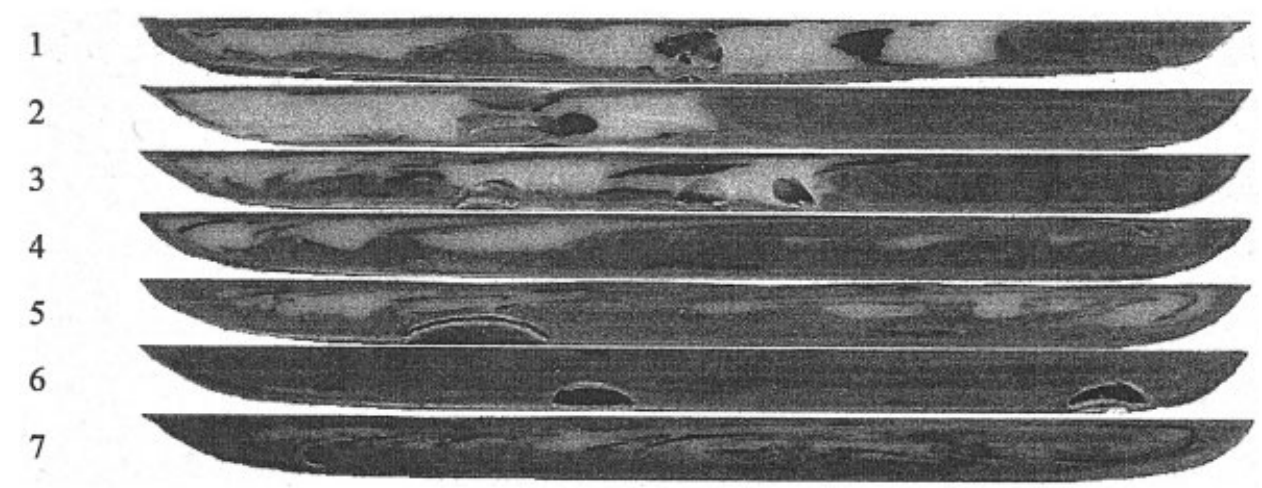

(c)

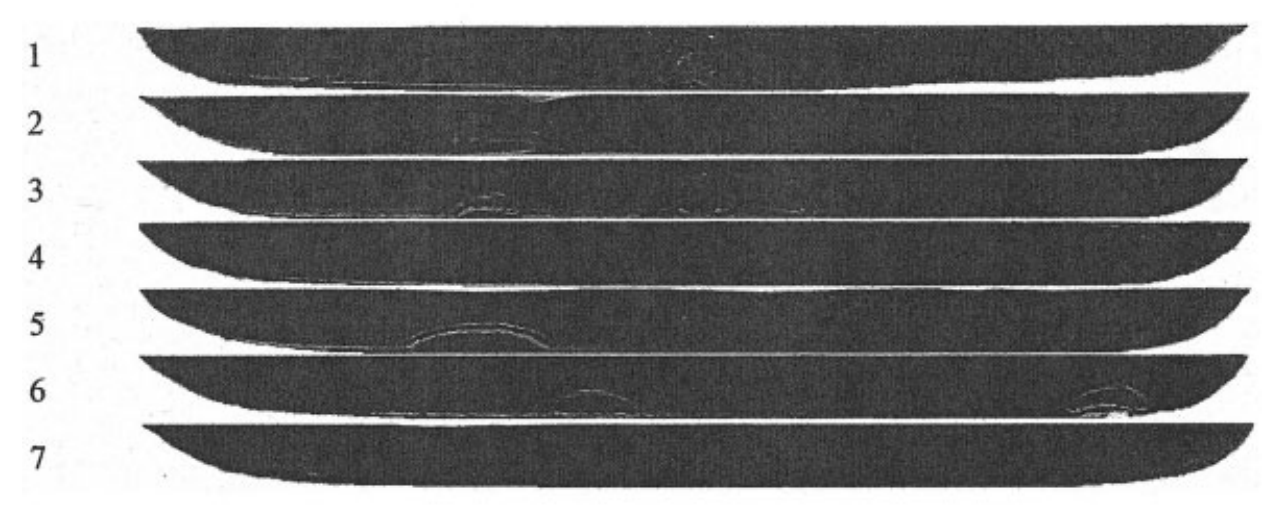

(d)

FIG. 2. (a)-(d): Grayscale and RGB monochromatic intensity maps of analyzed cuts. a, Gray; b, red channel; c, green channel; d, blue channel. 


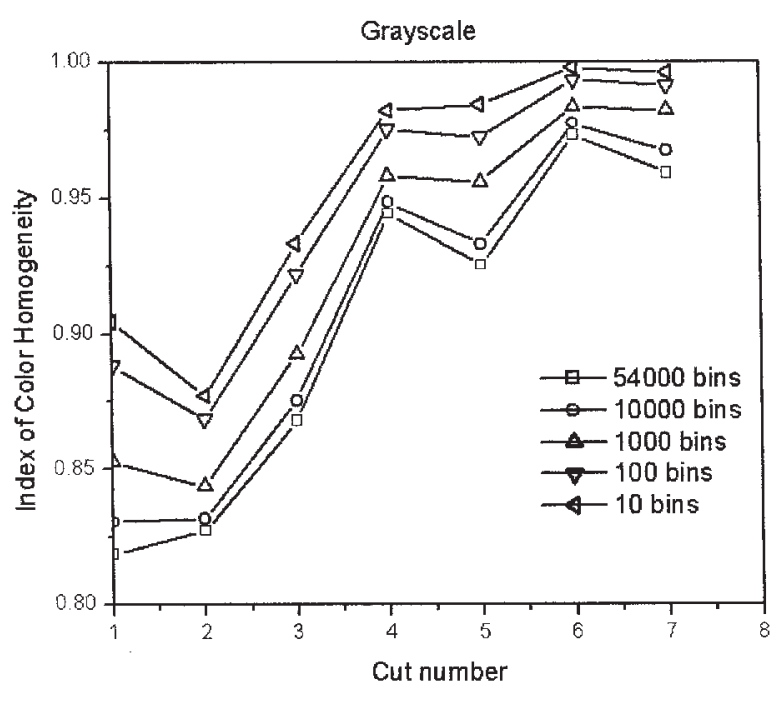

(a)

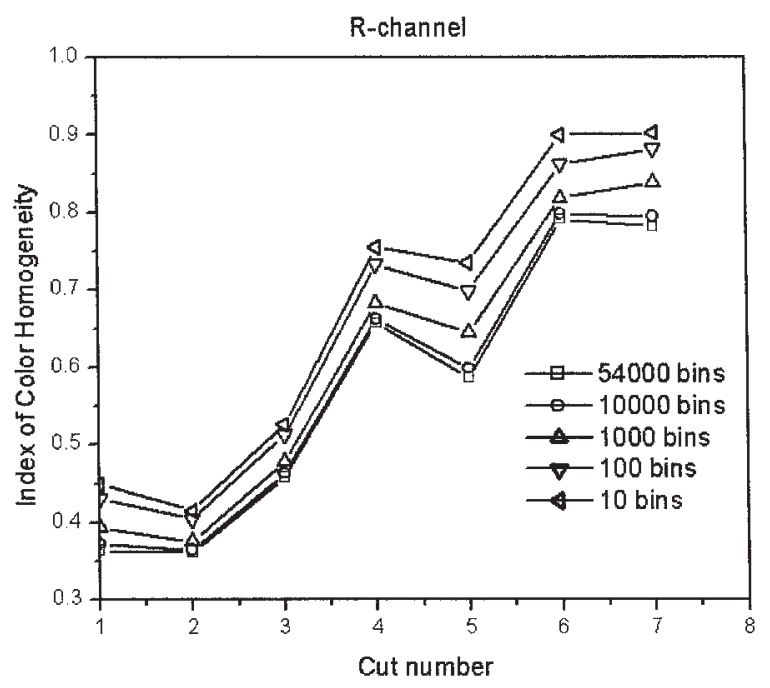

(b)

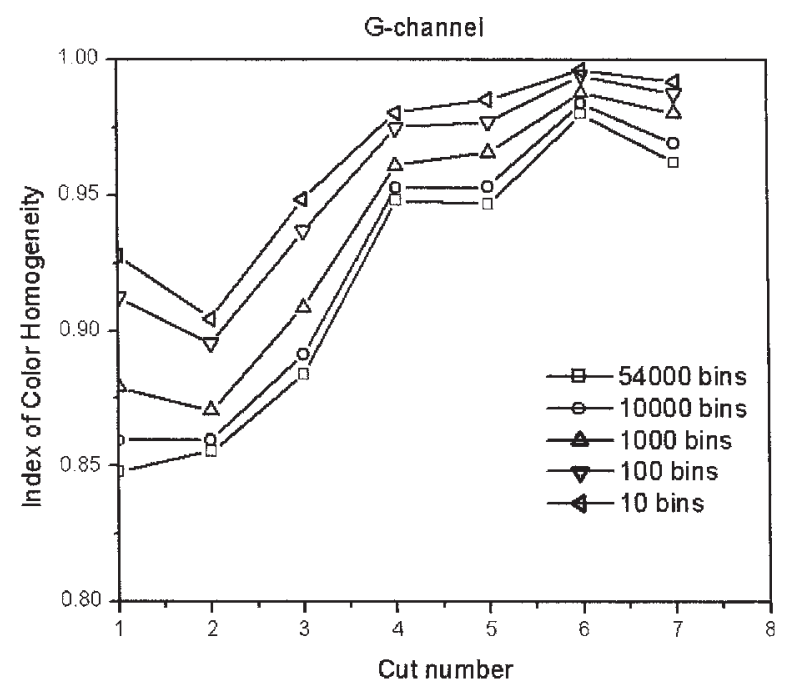

(c)

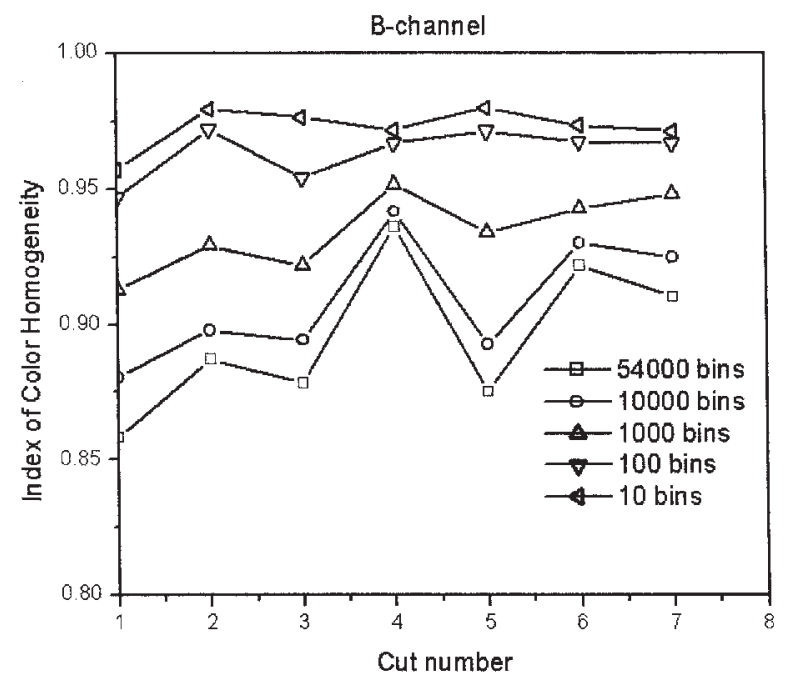

(d)

FIG. 3. (a)-(d): Evolution of color homogeneity index based on the grayscale and RGB entropic analysis. a, Based on grayscale; b, based on red channel; c, based on green channel; d, based on blue channel.

larger scales of observation (10 to 1000 bins). At the large scales, the overall distribution of bright/dark regions appears to be quite uniform for cuts \#4 through \#7. Another interesting observation is the slight decline of the index at the 2 nd cut depicted at large scales of observation. This in fact reflects that the large bright portions are more evenly distributed throughout the first image than they are for the second one.

In general, although the evolution of the index of color homogeneity along the extruder line reflects the correct trend of color upgrading, the analysis shows an overall low sensitivity in depicting slight changes in the quality of the images, because the overall deviations of intensity at the grayscale level are not so pronounced.

The color homogeneity measurements based on the intensities of the split RGB channels provide more opportu- nity in the selection of a higher sensitivity analysis. In the example taken here, with a color scheme based on mostly yellow and green, the red channel provides better sensitivity (higher contrast). The intensity deviations of the red channel are more pronounced when moving between yellow (high intensity of red) and green (low intensity of red) colors. Indeed, the intensity maps for the red channel depicted in Fig. 2b support this notion.

Because the contrast between bright and dark regions is more pronounced on the red channel, the sensitivity of the color homogeneity index increases, as shown in Fig. 3b. The overall evolution of the index along the extruder length shows similar trends with the analysis based on the grayscale images.

To complete the analysis, we computed the indices of color homogeneity for the green and blue channels, and the 
results are shown in Fig. $3 \mathrm{c}$ and d. In the experiment presented here, the green channel map is apparently not much different from the grayscale representation of the pictures. Overall, the green channel is slightly brighter than the grayscale one and shows some less-marked intensity deviations, which translates into slightly less sensitivity for the index of color homogeneity with an analogous evolution trend.

In contrast with all the previous observations, the evolution for the blue channel-based index appears to be quite flat from the 1 st to the last cut. The blue channel does not contribute significantly to the color images in the green/ yellow palette. In fact, all 7 cuts show quite low, evenly distributed intensity of blue (Fig. 2d). The plunge of the index at the 5th cut can be explained by a slightly higher overall intensity of this cut as compared with the other ones. This may very well be an artifact in the analysis. At this point it is important to stress that to minimize artifact, one should consider using a photo cell with standardized light conditions and a polarizing filter when taking the pictures for analysis. It is also possible to exclude the undesirable dependence on external light conditions in the analysis by using HIS (hue-intensity-saturation) maps rather than RGB maps.

\section{CONCLUSIONS}

In this work we propose a method of quantitative analysis for distributive mixing in polymer extrusion based on an entropic measure of color homogeneity for the extrudate. We illustrate the method by assessing the dynamics of distributive mixing in an industrial single screw extruder used to mix yellow and blue concentrates of ABS resin. The entropy measures rigorously the absolute quality of mixing achieved at a given scale of observation.

While the technique requires further tuning (such as better ways to chose the ideal combinations of intensities in the grayscale or split RGB channels analysis, as well as a standard shooting procedure for taking the color images), the method offers a quantitative, inexpensive way for industrial mixing quality control and optimization.

\section{ACKNOWLEDGMENTS}

The authors would like to acknowledge Dow Chemical Co. for providing technical assistance in the color mixing experiments and Ohio Supercomputer Center for the use of computing facilities. We have benefited from helpful discussions with Marco Camesasca.

\section{REFERENCES}

1. B.H. Maddock, SPE J., 15(5), 383 (1959).

2. H. Benkreira, R.W. Shales, and M.F. Edwards, Int. Polym. Proc., 7, 126 (1992).
3. M.A. Spalding and K.S. Hyun, Proceedings of the 61st Annual Technical Conference of the Society of Plastics Engineers, Nashville, TN, 229 (2003).

4. C. Maillefer, British Patent 964,428 (1964).

5. C. Maillefer, U.S. Patent $3,358,327$ (1967).

6. B.H. Maddock, SPE J., 23(7), 23 (1967).

7. G. LeRoy, U.S. Patent $3,486,192$ (1969).

8. C.I. Chung and R.A. Barr, U.S. Patent, 4,405,239 (1983).

9. J.D. Frankland, U.S. Patent 4,639,143 (1987).

10. Z. Tadmor and I. Klein, Engineering Principles of Plasticating Extrusion, Krieger Publishing Co., Malabar, FL (1978).

11. G. Lidor and Z. Tadmor, Polym. Eng. Sci., 16, 450 (1976).

12. R.T. Fenner, Polymer, 18, 617 (1977).

13. H. Lappe and H. Potente, Proceedings of the 41st Annual Technical Conference of the Society of Plastics Engineers, Chicago, IL, 174 (1983).

14. C. Rouwendaal, Proceedings of the 43rd Annual Technical Conference of the Society of Plastics Engineers, Washington, D.C., 30 (1985).

15. M. Gupta and T.H. Kwon, Polym. Eng. Sci., 30, 1420 (1990).

16. R.A. Barr and C.A. Chung, U.S. Patent 3,487,503 (1970).

17. R.V. DeBoo and C.B. Heard, U.S. Patent 3,762,693 (1973).

18. W.G. Yao, K. Takahashi, K. Koyama, and G.C. Dai, Chem. Eng. Sci., 52, 13 (1997).

19. W.G. Yao, S. Tanifuji, K. Takahashi, and K. Koyama, Polym. Eng. Sci., 41, 908 (2001).

20. T.H. Lee and T.H. Kwon, Adv. Polym. Tech., 18, 53 (1999).

21. G.M. Gale, U.S. Patent 4,419,014 (1983).

22. G.M. Gale, Proceedings of the 41st Annual Technical Conference of the Society of Plastics Engineers, Chicago, IL, 109 (1983).

23. R. Brzoskovski, J.L. White, W. Szydlowski, N. Nakajima, and K. Min, Int. Polym. Proc., 3, 134 (1988).

24. Y. Jabushida, R. Brzoskovski, J.L. White, and N. Nakajima, Int. Polym. Proc., 4, 219 (1989).

25. F.E. Dulmage, U.S. Patent 2,753,595 (1956).

26. R.L. Saxton, U.S. Patent 3,006,029 (1961).

27. W.L. Kruger, Plast. Eng., 37(10), 35 (1981).

28. C. Rauwendaal, Polymer Extrusion, Hanser Publishers, Munich (1986).

29. W. Michaeli, Extrusion Dies: Design and Engineering Computations, Hanser Publishers, Munich (1984).

30. Z. Tadmor and C.G. Gogos, Principles of Polymer Processing, John Wiley \& Sons, New York (1979).

31. P.V. Danckwerts, Appl. Sci. Res. A, 3, 279 (1953).

32. J.M. Ottino, The Kinematics of Mixing: Stretching, Chaos, and Transport, Cambridge University Press, Cambridge, UK (1989).

33. H.H. Yang, T. Wong, and I. Manas-Zloczower, "Flow Field Analysis of a Banbury Mixer," in Mixing and Compounding of Polymers, I. Manas-Zloczower and Z. Tadmor, editors, Hanser Publishers, Munich-Vienna-New York, 189 (1994).

34. T. Avalosse, Macromol. Symp., 112, 91 (1996).

35. W. Wang, I. Manas-Zloczower, and M. Kaufman, Int. Polym. Proc., 16, 315 (2001). 
36. W. Wang, I. Manas-Zloczower, and M. Kaufman, AIChE J., 49, 1637 (2003).

37. D.F. Styer, Am. J. Phys., 68, 1090 (2000).

38. A. Brandenberger, V. Mukhanov, and T. Prokopec, Phys. Rev. D: Part. Fields, 48, 2443 (1993).

39. A.O. Schmitt, H. Herzel, J. Theor. Biol., 188, 369 (1997).

40. Y. Zimmer, R. Tepper, and S. Akselrod, Ultrasound Med.Biol., 22, 1183 (1996).

41. R. Smolikova, M.P. Wachowiak, and J.M. Zurada, Comput. Biol. Med., 34, 355 (2004).

42. T.C. Devezas, and J.T. Corredine, Tech. Forecast. Soc. Change, 69, 317 (2002).

43. J. Hale, J. Psycholinguistic Res., 32, 101 (2003).

44. A. Golan, G. Judge, and L. Karp, J. Econ. Dyn. Control, 20, 559 (1996).

45. R.C. Campbell and R.C. Hill, Economics Lett., 64, 263 (1999).
46. J.D. Phillips, P.A. Gares, and M.C. Slattery, Land. Ecol., 14, 197 (1999).

47. P.O. Bodun, S. Shibusawa, A. Sasao, K. Sakai, and H. Honaka, J. Terramechanics, 37, 3 (2000).

48. H.D. Cheng, C.H. Chen, H.H. Chiu, and H. Xu, IEEE Trans. Image Process., 7, 1084 (1998).

49. H.D. Cheng, X.H. Jiang, and J. Wang, Patt. Recog., 35, 373 (2002).

50. N. Tavakoli, J. Vis. Comm. Image Rep., 4, 271 (1993).

51. C.E. Shannon, Bell Syst. Tech. J., 27, 379 (1948).

52. A. Ya. Khinchin, Mathematical Foundations of Information Theory, Dover Publications, New York (1957).

53. K. Alemaskin, M. Camesasca, I. Manas-Zloczower, M. Kaufman, E.K. Kim, M. Spalding, W.A. Trumbull, and R.D. Swain, Proceedings of the 62nd Annual Technical Conference of the Society of Plastics Engineers, Chicago, IL, 167 (2004).

Post-print standardized by MSL Academic Endeavors, the imprint of the Michael Schwartz Library at Cleveland State University, 2017. 